A N N A L E S Annales de Bretagne et des Pays de l'Ouest

\title{
La recherche à l'université de Bretagne occidentale (Brest) de 1995 à 2002
}

\section{(2) OpenEdition \\ Journals}

Édition électronique

URL : http://journals.openedition.org/abpo/1407

DOI : 10.4000/abpo.1407

ISBN : 978-2-7535-1491-1

ISSN : 2108-6443

\section{Éditeur}

Presses universitaires de Rennes

\section{Édition imprimée}

Date de publication : 20 octobre 2003

Pagination : $143-160$

ISBN : 978-2-86847-929-7

ISSN : $0399-0826$

\section{Référence électronique}

"La recherche à l'université de Bretagne occidentale (Brest) de 1995 à 2002 », Annales de Bretagne et des Pays de l'Ouest [En ligne], 110-3 | 2003, mis en ligne le 20 octobre 2005, consulté le 15 septembre 2020. URL : http://journals.openedition.org/abpo/1407 


\section{La recherche à l'université de Bretagne occidentale (Brest) de 1995 à 2002}

Voici la liste, aussi exhaustive que possible, des travaux de recherche historique menés de 1995 à 2002 dans le cadre du département d'histoire de la Faculté des Lettres et Sciences Sociales VictorSegalen de l'université de Bretagne occidentale à Brest (UBO). Ce recensement s'appuie en premier lieu sur l'important travail de Marie-Rose Prigent et de Brigitte Pennec portant sur les mémoires de maîtrise d'histoire déposés et consultables à la bibliothèque du Centre de Recherche Bretonne et Celtique (CRBC, UMR 6038 du CNRS, 20 rue Duquesne, 29285 Brest) et portant sur l'histoire de la Bretagne. Ce sont les normes de présentation de ce fichier que nous avons adoptées. Ce travail a été complété par l'inventaire des travaux de recherche en histoire contemporaine soutenus à l'UBO de 1966 à 2000 dressé par Yvon Tranvouez, d'abord publié dans Kreiz 7, «Études sur la Bretagne et les Pays Celtiques ", 1997, p. 181-200 puis complété en février 2001. Pour les thèses et les DEA portant sur les autres périodes (informations communiquées par Françoise Maurel), ainsi que pour les maîtrises non déposées ou ne concernant pas le champ de recherche du CRBC, nous avons sollicité le concours des enseignants et dépouillé les registres officiels de la Faculté. Pour cette raison, les informations sont parfois moins complètes (pagination, illustrations). Sans vouloir en analyser le contenu, cet inventaire montre bien le dynamisme, l'ampleur, la diversité et la richesse de la recherche historique à l'Université de Bretagne occidentale. Puisse ce travail des étudiants, des chercheurs et des enseignants-chercheurs être connu et utile.

Christian BOUGEARD

\author{
Habilitations à diriger \\ des recherches
}

BouthILLON, Fabrice, L'ironie de l'histoire. Épistémologie de l'histoire et phénomène totalitaire, 2002; dir. Y. TRANVOUEZ.

CASSARD, Jean-Christophe, Les Bretons dans le millénaire médiéval : approches sociétales, 2000; dir. J. KERHERVE.

\section{Doctorats nouveau régime}

PAUBERT, Laurent, Formation de l'opinion publique, politisation et structuration des identités politiques en Bretagne au XIX ${ }^{e}$ siècle. Finistère-Morbihan (18481914), 2003; dir. Ch. BOUGEARD.

CARICHON, Christophe, Le scoutisme catholique en Bretagne des origines aux années 1970, 2002; dir. Y. TRANVOUEZ.

Coativy, Yves, La monnaie bretonne de l'an mil à 1499, 2000; dir. J. KERHERVE.

Gallice, Alain, Guérande et le pays guérandais entre 1340 et 1540, 2000; dir. J. KERHERVE.

LE GoIc, Pierre, Brest en reconstruction : mythes, acteurs et rythmes d'une renaissance. "Antimémoires " d'une ville (1794-1994), 2000; dir. Y. TRANVOUEZ.

MARTIN, Jean, La manufacture des toiles "Bretagnes " (1670-1830), 1997; dir. J. TANGUY.

LE PAGE, Dominique, L'administration financière de la Bretagne de la fin $d u X V^{e}$ siècle aux années 1540. Analyse du processus d'intégration d'une principauté au royaume, 1995 ; dir. J. KERHERVE. 


\section{Diplômes d'Études Approfondies (DEA)}

\section{2}

AbOllivier, Philippe, Les monnaies de la cité des Osismes, dir. G. MoITrIEuX.

Blin-PRIJAC, Claire, L'habitat déserté de Goaremm ar c'hoz Tier en PlonéourMénez. État de la recherche et travaux de préparation à une fouille archéologique, dir. J.-C. CASSARD.

BRES, Arnaud, Mémoire du mouvement républicain " provisoire » nord-irlandais, de 1969 à nos jours, dir. Ch. BOUGEARD.

BRUnET, Gaëtan, Les Guerches dans l'Ouest de la France au haut Moyen Âge, dir. J.C. CASSARD.

CARNEY, Sébastien, Résurrection. La Grande Guerre de "Breiz Atao », 1919-1939, dir. Ch. BOUGEARD.

HENRY, Christelle, La naissance du cyclisme en Bretagne, dir. Y. TRAnvouEz.

LANNUZEL, Yannick, Biographies et prosopographie des Bretons en Indochine (fin XVIII ${ }^{e}$-XIXe siècle), dir. Y. TranvouEz, Ph. BRUNET.

LEPLANT-DELAUNAY, Christine, La femme indochinoise sur papier, un être idéal?, 2002 ; dir. Y. Tranvouez, Ph. Brunet.

MARLIER, Jean-Noël, Les fournisseurs de la Marine sous le Second Empire, dir. Y. TranVOUEZ.

RIVIERE, Christophe, Approche de la légitimité politique des notables bretons. Éléments pour une étude des conseillers généraux de Bretagne (1919-1958), dir. Ch. Bougeard.

Rolland, Yves et Rolland-L'HELgOUAlC'H, Hélène, Identité locale et frontières communales dans l'arrondissement de Châteaulin, 1800-1950, dir. Y. TRANVOUEZ, J.-Y. CARLUER.

SPARFEL, Yohann, Géographie des sites funéraires du néolithique à l'âge $d u$ bronze moyen. Les exemples du nordouest du Léon et du Pays Bigouden, dir. P. GOUlETQUER.

TrABUT, Aline, Les immigrés français et leurs descendants en Suède de 1685 à 1818. Modes de vie, mentalités et formes d'intégration à la société suédoise, dir. F. ROUDAUT.

\section{1}

Chamelot, Marie-Noëlle, La mer et les marins dans les chroniques du temps de la duchesse Anne de Bretagne, dir. J. KERHERVE.

GuENADOU, Éric, Les milices garde-côtes de l'amirauté de Quimper au XVIII siècle (1716-1792), 2001; dir. F. RoudAuT.

JAIN, Benoît, Pierre Jaïn, sculpteur à Kerlaz, dir. Y. TRANVOUEZ.

KERMOAL, Emmanuelle, L'Action catholique en milieu rural des origines à 1975 dans l'Evêché de Quimper et de Léon, dir. Y. TRANVOUEz.

MADEC, David, Les fermes modèles au XIXe siècle. Modalités, acteurs et enjeux, dir. Y. TRAnvouEz.

\section{0}

Blanc-Bignand, Annie, Gabriel Couppé de Kervennou, bourgeois lannionnais, homme politique breton, dir. F. ROUDAUT.

MoAL, Laurence, La vision des étrangers dans les chroniques bretonnes, dir. J. KERHERVE.

Rotoloni, Céline, La sociabilité des gens de mer dans l'Amirauté de Saint-Brieuc de 1750 à 1815, dir. F. ROUDAUT.

\section{9}

BERNARD, Norbert, Chemins et structuration de l'espace en Cornouaille $d u$ $v^{e}$ siècle à la fin du XVI⿸ siècle. Exemples autour de la commune d'Ergué-Gabéric, dir. J. KERHERVE.

Chevalereau, Fabienne, Les Finistériens et la guerre d'Algérie, de 1954 à nos jours, dir. Ch. BOUGEARD.

Le Malefan, Serj, La Seconde république dans les Côtes-du-Nord, dir. Ch. BOUGEARD.

PAUBERT, Laurent, L'évolution politique de l'Ouest breton de 1848 à 1940. Élites et suffrage universel en Finistère et Morbihan, dir. Ch. BOUGEARD.

\section{8}

BESSELIEVRE, Jean-Yves, Les bombardements de Brest (1940-1944). Stratégies alliées, riposte allemande et réaction des populations, 1998; dir. Y. TRANVOUEZ. 
DefaIx, Jérôme, Céramique et acculturation en Armorique aux er $^{\text {et }}$ It $^{e}$ siècles, dir. M.-Cl. L'HUILLIER.

DERRIEN, Dominique, Tanneurs, tanneries et activités connexes dans le Finistère, de la fin de l'Ancien Régime à la Première Guerre mondiale, dir. Y. TRANVOUEz.

KERNEVEZ, Patrick, Les mottes castrales du Finistère : de l'inventaire archéologique à l'interprétation historique, dir. J. KERHERVE.

LE BLOAS, Alain, L'administration du département et des districts du Finistère (17901793), dir. F. ROUDAUT.

LE GALl, Laurent, La Seconde République et les campagnes du Finistère, dir. Y. TRANVOUEZ.

LE SEAC'H, Emmanuelle, Les ateliers de sculpteurs sur pierre en Bretagne, $X V^{e}$ et XVII siècle, dir. F. ROUDAUT, J.-Y. ÉVEILLARD.

YvEn, Estelle, Mésolithique et Néolithique dans le Trégor finistérien, dir. P. GOULETQUER.

\section{7}

BARGAIN, Frédéric, Un humaniste breton au XIX $X^{e}$ siècle : Auguste Dupouy, dir. J. KERHERVE.

LAHELLEC, Philippe, Albert le Grand de Morlaix : sources et méthodes, dir. J. KERHERVE, B. TANGUY.

\section{6}

BERRIVIN, Antoine, La dévisibilation du catholicisme : l'exemple du diocèse de Quimper et de Léon, de la fin des années 1950 aux années quatre-vingts, dir. Y. TRANVOUEZ.

BANCEL-CHARLES, Isabelle, Robert d'Arbrissel et le christianisme celtique, dir. J. KERHERVE.

COCHARD, Marc, L'office de capitaine urbain au $X^{v}$ siècle, d'après les lettres et mandements des ducs de Bretagne, dir. J. KERHERVE.

GesLin, Mona, Vie politique des localités rurales du Sud-Ouest de la Cornouaille, 1750-1850, dir. F. RouDAuT.

Le Dez-GrafFin, Fabienne, La population brestoise dans la seconde moitié du XIX siècle, dir. Y. TRANVOUEZ.
MORVAN, Frédéric, Les gens de guerre du Duché de Bretagne de 1294 à 1491. Étude du "Livre des arts" (1294). Identifications, dir. J. KERHERVE.

\section{5}

Contivy, Yves, La noblesse léonarde au $X v^{e}$ siècle, dir. J. KERHERVE.

CARICHON, Christophe, Le scoutisme en Bretagne des origines à nos jours, dir. Cl. GESLIN.

Cloarec, Denis, La presse catholique du diocèse de Quimper et de Léon, XIXe$X X^{e}$ siècle : "Une industrie du zèle ", dir. Y. TRANVOUEZ.

DREYER, Jean-François, Le rentier de Châteaulin (1543-1545), dir. J. KERHERVE.

LEMAIRE-BERTHO, Laure, La prédication en langue française en Basse-Bretagne sous l'Ancien Régime, dir. F. Roudaut.

LE GoIc, Pierre, Brest en reconstruction, ou l'instant durable. Acteurs, rythmes, représentations d'une renaissance, dir. Y. TRanvouez.

PichouRon, Patrick, La noblesse trégoroise (vers 1380-vers 1550). L'exemple des seigneurs de Coëtmen et de Tonquédec, dir. J. KERHERVE.

Salaun, Armelle, Vitrail et société dans le diocèse de Quimper et de Léon, de 1860 à nos jours, dir. Y. TRANVOUEZ.

\section{Mémoires de maîtrises}

\section{2}

ABIVEn, Stéphane, La vie religieuse à Plouguerneau du Concordat à 1914, dir. M.-T. ClOITRE.

Boulat, Guillaume, France et Belgique dans la politique des compensations à l'époque de Napoléon III, dir. F. BouthILLON.

Chenet, Laurent, Prosper Levot ou l'élaboration de la production historique d'un érudit du XIXe siècle : d'après sa correspondance, dir. M.-T. CLOITRE.

Coquil, Christophe, Le Conquet du paléolithique à la période romaine, dir. J.Y. ÉVEILLARD.

CREN, Johnny, Guipavas de 1643 à 1820. Naître, s'unir et mourir dans un village breton à l'époque moderne, dir. J.-L. LE CAM. 
Delangle, Caroline, La symbolique des ducs de Bretagne et la Maison de Dreux, dir. Y. COATIVY.

DREAU, Kristell, L'enseignement primaire public à Quimper de 1880 à 1902, dir. M.-T. CLOITRE.

Eymann, Olivier, Etre fou au XIXe siècle : les internés d'office du Finistère de 1826 à 1861, dir. J.-Y. CARLUER.

FALCHIER, Yohann, Ar Falz : d'une idée laïque à une idée socialiste et régionaliste (1945-1965), dir.?

FALHUN, Isabelle, Les changements techniques et culturels vécus dans le Morbihan rural de 1950 à 1970, dir. Y. TRANVOUEZ.

Gloanec, Sophie, Poétique de Monseigneur Duparc, dir. Y. TRANVOUEZ.

GUIVARCH, Laëtitia, Les tombeaux bretons de la collection Gaignières, dir. J. KERHERVE.

KERDONCUFF, Isabelle, Étude sociale des gens de justice à Landerneau au XVIII siècle (1700-1789), dir. F. ROUDAUT.

LAGADEC, Cédric, La délinquance à Brest pendant la Monarchie de Juillet (1830 1848), dir. J.-Y. CARLUER.

LAOT, Albert, Chronique d'une famille rurale léonarde : essai d'une généalogie appliquée, dir. F. RoudAuT.

LE BELlec, Mickaël, Délinquance et criminalité dans l'arrondissement de Lannion au XIXe siècle (1849-1899) à travers le "Lannionnais ", dir. J.-Y. CARLUER.

LE GoFF, Marc, Autour des arts : le discours d'un hebdomadaire catholique : l'Écho Paroissial de Brest (1898-1939), dir. M.Th. CloITRE.

LE MAITRE, Bleuenn, La famille et la seigneurie de Kergroadez : 1400-1560, dir. Y. COATIVY

MARCHAND, Christophe, L'octroi à Quimper au XIXe siècle, dir. J.-Y. CARLUER.

MAZEAS, Mariana, Les chapelles SainteBarbe et Saint-Fiacre du Faouët, $X V^{e}$-XVt siècles : histoire, société, art, dir. J. KERHERVE.

Menard, Philippe, Théodore Le Hars : les étapes d'une vie, 1861-1928, dir. M.-Th. CLOITRE.

PICARD, Marine, La droite et l'extrême droite dans le Finistère du début de la guerre
d'Algérie aux élections présidentielles de 1969, dir. Ch. BOUGEARD.

PICHON, Marie-Laure, La vie politique du canton de Lesneven : 1945-1971, dir. Ch. BOUGEARD.

Pluchon, Frédéric, La réception du cheval d'orgueil de Pierre Jakez Hélias, dir. Y. TRanvouez.

PRAT, Stéphane, Les élections législatives de 1958, 1962 et 1967 dans le Finistère, à travers les journaux et les archives, dir. Ch. BOUGEARD.

SAllou, François, Répertoire d'un fonds d'archives du château de Kergrist en Ploubezre, Côtes-d'Armor, dir. J. KERHERVE.

TANGUY, Nicolas, Les chapelles du Finistère au XIX siècle d'après les enquêtes diocésaines (État, religion, société), dir. M.-T. CLOITRE.

TANNE, Erwann, L'Histoire de Bretagne en bandes dessinées de la Préhistoire à 1532 : étude sur les sources historiques et leur mise en page. Vers un nouveau rapport de la $B D$ à l'histoire?, dir. J.-C. CASSARD.

UguEn, Guillaume, Les relations francobavaroises de 1870 à 1934, dir. F. BOUTHILLON.

\section{1}

ABILIOU, Anne-Laure, Société et vie quotidienne à Plouguerneau au XVIII siècle d'après les inventaires après décès, dir. F. ROUDAUT.

AUTRET, Stéphane, Les généalogies d'Anne de Bretagne. L'histoire au service des ducs, dir. J-C. CASSARD.

CAILlEAU, Marlène, Le bal à Brest de 1870 à 1914, dir. M.-T. CLOITRE.

Chiroussot, Emeric, L'amour dénaturé des crimes familiaux dans le Finistère de 1870 à 1900, dir. J.-Y. CARLUER.

COADOU, Jean-Benoît, L'édition brestoise de la "Gazette " et la guerre de succession d'Espagne 1708-1714: outil de propagande au service du pouvoir monarchique ou vecteur d'informations locales et globales d'une société française et européenne en devenir, dir. J.L. LE CAM. 
CORRE, Nicolas, Un monde en mutation : l'Achaïe, la Thessalie et la Macédoine de Dioclétien à Théodose II (284-450), dir. B. LAN²ON.

Costiou, Laëtitia, Les fontaines du Pays Glazik, dir. J.-Y. ÉVEILLARD.

Crouan, Erwan, Jean Crouan 1906-1985. Un Finistérien dans la tourmente du siècle, dir. Ch. BOUGEARD.

Dathanat, Pierre, L'UNR et les Gaullistes dans le Finistère de 1953 à 1969, dir. Ch. BOUGEARD.

DELIN, Alexandre, L'insurrection girondiste du département du Finistère 1793-1794, s.l., s.n., 2001, 130 p.; dir. A. de MATHAN.

DupuY, Julie, Les fêtes médiévales contemporaines en Bretagne, dir. J.-C. CASSARD.

GloEnec, Anne, Dostoïevski face à L'Occident, dir. Ph. BRUNET.

Guichaoua, Lise-Marie, La société de secours mutuel de Lesneven : un siècle d'histoire 1857-1958, dir. M.-T. CLOITRE.

Gueguen, Isabelle, Châteauneuf-du-Faou dans la seconde moitié du XIX siècle (1850-1914), dir. M.-T. CloITRE.

GOURMELON, Gérard, Regards croisés $d u$ $F L B$. Les fronts de libération de la Bretagne et leur médiatisation entre 1966 et 1981, dir. Y. TRANVOUEZ.

HAMONIC, Nadège, Le rayonnement de l'abbaye Saint-Aubin d'Angers (XI'-XII siècles), dir. Y. HILLION.

HARDY, Bertrand, Les maîtres d'école dans les biographies de sermons funèbres luthériens : professionnalisation et confessionnalisation en Allemagne au XVII siècle, dir. J.-L. LE CAM.

Helias, Périg, La représentation de la Bretagne à travers le Pathé Journal (1910-1970), dir. Y. TRANVOUEZ.

HENRY, Christelle, Le cyclisme féminin breton 1897-2001, dir. Ph. BRUNET.

IBANEZ, Marlène, Le bal à Brest de 1870 à 1994, dir. M.-T. CLOITRE.

KERVRAN, Sophie, La protection du patrimoine finistérien au XIXe siècle (17901913) : des monuments historiques aux sites naturels, dir. M.-T. CloITRE.

LAGADEC, Philippe, L'usage du monde de Nicolas Bouvier. Une fascination de l'Orient, dir. Ph. BRUNET.
LAOT, Gwenaëlle, la Révolution Culturelle Jaciste. Aspects culturels de la JAC/F dans le Finistère (1945-1960), dir. Y. TRANVOUEZ.

LaUtrou, Annaïg, Les minihis, dir. J.-C. CASSARD.

LAz, Virginie, Transcription et étude du registre des lettres scellées à la Chancellerie de Bretagne en 1513, dir. J. KERHERVE.

LE DALL, Olivier, "On prie, vous souffrez, on les aura ». La Grande Guerre au miroir du Patro, bulletin des patronages de Ploudalmézeau, dir. Y. TRANVOUEz.

LE GALL, Olivier, La Seconde Guerre mondiale dans le canton de Carhaix, dir. Ch. BOUGEARD.

LE GALL, Olivier, Les monuments aux morts de la première guerre mondiale. Étude sur les cantons de Daoulas, Brest, Landerneau et Guipavas, dir. M.T. CLOITRE.

LE GOUAREGUER, Mikaël, Les procureurssyndics et miseurs de Morlaix au XVII siècle, dir. F. ROUDAuT.

LE GuERn, Cédric, Médecins, chirurgiens et apothicaires de la ville de Brest au siècle des Lumières, dir. Ph. JARNOUX.

LE MOAL, Denis, Le service du travail obligatoire (STO) dans le Finistère pendant la Seconde Guerre mondiale, dir. Ch. BOUGEARD.

LE MoAL, Solenn, La politique à Cadiz dans les années 1980, dir. Ch. BougEARD.

LE PAPE, Lucie, Les fontaines du sud-est du pays Bigouden : Tréméoc, CombritSainte-Marine, Pont-l'Abbé, PlobannalecLesconil, Loctudy, dir. J.-Y. ÉVEILLARD.

LEPLANT, Christine, L'imaginaire colonial en Indochine à travers les affiches, dir. $\mathrm{Ph}$. BRUNET.

LUGUERN, Béatrice, L'enfance dans les sermons luthériens, dir. J.- L. LE CAM.

MADEC, François, Le bureau de bienfaisance de Brest (1914-1941), dir. M.-T. CloITRE.

MOREAU, Régis, Les évolutions politiques à Quimper de la Libération à l'aube des années 70, dir. Ch. BOUGEARD.

Morvan, Kilian, "Kroaz ar Vretoned", 1898-1920. Un journal des Côtes-du-Nord entre paysanisme français et catholicisme breton, dir. M.-T. CLOITRE. 
NOURRY, Jérôme, Le bureau de bienfaisance de Quimper 1817-1914, dir. M.-T. Cloitre.

NovaK, Cédric, Ernst Jünger et son temps, dir., F. BOUTHILLON.

OLIVIER, Serge, La police à Brest (18151848), dir. J.-Y. CARLUER.

PorTIER, Sébastien, Cirques et gladiature dans l'Occident romain de Constantin à Honorius (306-423 après J.-C.), dir. B. LANCON.

QUEMENER, Loïc, Le sport dans La Dépêche de Brest \& de l'Ouest, 1932-1938, dir. Y. TRANVOUEZ.

Rolland, Yves et L'Helgoualc'H, Hélène, Identités et frontières communales dans la Basse-Vallée de l'Aulne, 1800-1950. Châteaulin, Port-Launay, Saint-Ségal, Pont de Buis-lès-Quimerc'h, dir. J.-Y. CARLUER.

ROUILLER, Yann, Les ouvriers de l'arsenal de Brest pendant la guerre de sept ans (1756-1763), dir. Ph. JARNOUX.

Selino, Kristel, La découverte des Khmers rouges à travers la presse de 1975 à 1979, dir. Ph. BRunet.

SPARFEL, Yohann, Prospection et inventaire des mégalithes, tumulus, cairns et tertres dans l'ouest du Léon (FinistèreBretagne), dir. J.-Y. ÉvEILLARD.

STRUB, Philippe, Le préfet maritime et la reconstruction de la Marine du Ponant (du 19 septembre 1944 au premier semestre 1950), dir. Ch. BOUGEARD.

\section{0}

AUBLE, Hélène, L'invasion et l'occupation du Tibet par la Chine 1950-1959, dir. Ph. BRUNET.

BARON, Frédéric, Les congrès catholiques dans le diocèse de Quimper et de Léon (1880-1962). D’après la Semaine Religieuse de Quimper et de Léon, dir. M.-T. CLOITRE.

BARON, Nicolas, Sport et identitarisme, 1948-1998, dir. D. LE GUYADER.

BoDros, Laurent, “L'idée française » dans le fait colonial en Annam (1875-1911): à partir du fonds Groleau, dir. Ph. BRUNET.
Bony, Aurélien, La campagne de 1812 narrée par Tolstoï dans Guerre et Paix, dir. Ph. BRUNET.

BOURGES, Aziliz, Édition et commentaire historique de l'enquête inédite réalisée à Guingamp sur Charles de Blois (†1364), dir. J.-C. CASSARD.

BuRlion, Emmanuel, Brest au Second Empire : la vie religieuse (1852-1870), dir. M.-T. CloITRE.

CARIOU, Florence, L'immigration algérienne dans le Finistère de 1945 à 1962, dir. Ch. BOUGEARD.

CARlo, Anne-Lise, Analyse des manuels et programmes officiels d'instruction civique au collège de 1945 à 1985, dir. Ph. BRUNET.

ChafFron, Nicolas, Charles de Valois, dir. J. KERHERVE.

CHEVER, Sébastien, Étude du domaine royal de Conq-Fouesnant-Rospreden d'après le rôle-rentier de 1540, dir. J. KERHERVE.

Cocaign, Franck, Étude d'un périodique, le " Kannad ar Galoun Zakr », miroir de tout un catholicisme finistérien, dir. M.-T. CloITRE.

Colin, Philippe, L'armement brestois sous la Restauration, 1814-1830, dir. M.-T. CloITRE.

CORDEROCH, Vincent, Les prisonniers évadés finistériens (1940-1945): ces héros de l'ombre ou le refus de la captivité française ou allemande, dir. Ch. BOUGEARD.

CoRolleur, Christian, L'Abbé Le Moan et le Kannadick Plourin, 1914-1925, dir. M.-T. CloITRE.

DANIEL, Béatrice, Le canton de Plomeur pendant la Révolution (1789-1799), dir. J.-Y. LE CAM.

DuPONT, Anne, Architecture de l'âge du Fer en Armorique : état de la recherche et réflexion sur les bâtiments à poteaux plantés porteur de la charpente, dir. J.-Y. ÉVEILLARD.

ELY, David, "Uranus " : polémique sur l'épuration, dir. Ph. BRUNET.

GOARZIN, Véronique, Transcription et étude du registre $B 16$ des lettres scellées de la chancellerie de Bretagne en 1506, dir. J. KERHERVE. 
Gueguen, Yannick, Le commissariat de police de Quimper : 1900-1920, dir. J.Y. CARLUER.

GuENNOC, Pierre-Yves, La condamnation au bagne (1789-1794) : une révolution?, dir. Ph. JARNOUX.

GUINET, Bertrand, Les incendies criminels dans le Finistère : 1811-1900, dir. J.-Y. CARLUER.

HELLWINKEL, Lars, Brest, base navale de la Kriegsmarine (1940-1944), dir. Ch. BOUGEARD.

JACQ, Guillaume, Le Club Alpin Français de Brest (1969-1999), dir. Y. Tranvouez.

JAFFrE, Nolwen, Étude démographique Plouguerneau : 1728-1828. Les mutations sociales engendrées par la Révolution dans une paroisse mi-maritime mi-rurale, dir. F. Roudaut.

JaOUEN, Angélique, De la Caisse armoricaine de compensation à la Caisse d'allocations familiales du Nord-Finistère (1921-1955), dir. Ch. BOUGEARD.

JEGOU, Christophe, Le patronat dans le SudFinistère de 1860 à 1914, dir. J.-Y. CARLUER.

Joncour, Cyrille, Les États de Bretagne et la guerre d'indépendance américaine 1778-1786, dir. Ph. JARNOUX.

LANNUZEL, Yannick, L'amiral de La Grandière, gouverneur de la Cochinchine, dir. Ph. BRUNET.

LE BERRE, Stéphane, Le domaine royal dans la région de Châteaulin d'après le rentier de 1544-1545, dir. J. HERHERVE.

LE Bот, Gwendal, Les relations entre Philippe Auguste et ses vassaux à travers " la Philippide " de Guillaume Le Breton. Un aperçu de l'idéologie royale au début du XII e siècle, dir. J. KERHERVE.

LE Bot, Nicolas, Les suicides dans le Finistère au XIX $X^{e}$ siècle, dir. J.-Y. CARLUER.

LE CORRE, Ewan, Robert d'Artois (12871342), dir. J. KERHERVE.

LE COz, Franck, Marx et Bakounine, ou l'action et la révolution en débat (18681875), dir. Y. TRANVOUEZ.

LE MAITRE, Éric, L'immigration espagnole dans le Finistère, 1936-1939, dir. Ch. BOUGEARD.

LE MARTRET, Rémy, Étude démographique de la paroisse de Perros-Guirec aux XVI, XVII et XVIII siècles, dir. R. LEPROHON.
LE NY, Christophe, Michelet, ou le quotidien de l'historien. Étude de la Correspondance générale, dir. Y. TRANVOUEZ.

LEBIGRE, Corinne, Aspects de la vie des Bretons au Havre, 1870-1914, dir. M.-T. CLOITRE.

LECUILLIER, Guillaume, Le château du Taureau au XVIII ${ }^{e}$ siècle : architecture, vie quotidienne, "Bastille morlaisienne ", dir. Ph. JARNOUX.

LEROY, Steven, L'opinion publique française et l'agression japonaise en Mandchourie, dir. Ph. BRUNET

LOISEAU, Sandrine, Étude des actes de Charles de Blois et Jeanne de Penthièvre, duc et duchesse de Bretagne (13411364). Un modèle de gouvernement princier, dir. J. KERHERVE.

LOURGOUILLOUX, Virginie, Société et vie quotidienne à Audierne et Esquibien au XVIII siècle d'après les inventaires après décès, dir. F. Roudaut.

MADEC, David, Les agglomérations secondaires dans la cité des Osismes et des Coriosolites, dir. J.-Y. ÉVEILLARD.

MEDARD, Loïc, Société et Mentalité sous la Révolution à Quimper (1785-1800), dir. J.- L. LE CAM.

MENEz, Valérie, Bertrand d'Argentré et le sentiment national breton, dir. J. KERHERVE.

MENOT, Anthony, La vision des colonies à travers les manuels scolaires, dir. Ph. BRUNET.

Mingant, Carine, La vie des Brestois dans une ville détruite de 1944 à 1947, dir. Ch. BOUGEaRD.

NIcoL, Marie-Ève, Le tribunal de commerce de Brest sous la Révolution, dir. Ph. JARNOUX.

NICOLAs-QuENET, Gaële, La vie quotidienne à l'hôpital psychiatrique de Quimper de 1938 à 1945, dir. Ch. BOUGEARD.

PARIS, Myleine, Bertrand d'Argentré et l'histoire de Bretagne, dir. J. KERHERVE.

Pelle, Jean Marie, "Le Semeur » et l'esclavage, dir. J.-Y. CARLUER.

PERON, Ludovic, Les conseillers généraux sous la Troisième République dans le Finistère (1871-1940), M.-T. Clortre.

PERREAU, Tristan, Les monuments aux morts de la guerre 1914-1918 dans l'ar- 
rondissement de Châteaulin, dir. M.-T. Cloitre.

POULPIQUET, Brigitte de, Vie et patrimoine des Gilart de Keranflec'h (1650-1900), dir. Ph. JARNOUX.

PRIJAC, Claire, Les habitats désertés médiévaux dans les Monts d'Arrée, état de la recherche et archéologie du paysage, dir. J.- C. CASSARD.

QUEMENEUR, Anne, Les intellectuels et le conflit yougoslave: analyses, représentations et polémiques dans la presse et l'édition française, dir. D. LE GUYADER.

Ribault, Serge, Les marins FNFL du Finistère. Étude sociologique, dir. Ch. BOUGEARD

SaluIC, Mathias, Les Échecs en Bretagne : 1921-2000, dir. Y. Tranvouez.

Samson, Gaelle, Un canton du Finistère dans la Seconde Guerre mondiale. Pont l'Abbé face à l'occupation allemande. 1939-1944, dir. Ch. BOUGEARD.

TANNEAU, Nicolas, L'opinion publique à Quimper durant la Première Guerre mondiale, Ch. BOUGEARD.

Tolson, Mélanie, Anne de Bretagne dans l'histoire et la litterature, dir. J. KERHERVE.

TомсZAK, Grégory, Étude des descriptions physiques des bagnards de Brest pour la période 1749-1789, dir. Ph. JARNOUX.

TREBAOL, Christophe, La Bretagne des rois aux ducs, du royaume indépendant à la principauté féodale, dir. J. KERHERVE.

\section{9}

Allain, Véronique, La paroisse de BourgBlanc à l'époque conciliaire : 1958-1972, dir. Y. TRANVOUEZ.

ARDINAT, Gilles, Wilhelm Reich et la question nazie. Autour de "La Psychologie de masse du fascisme ", dir. F. BOUTHILLON.

AUNE François, L'empire romain et l'Arménie (11 av., 224 apr. J.-C.), dir. B. LANCON.

BARnABe Magali, La colonie de SaintDomingue pendant la Révolution française (1792-1793) d'après les archives d'Adrien Nicolas de La Salle, dir. Ph. JARNOUX.

BLANCHARD, Valérie, La démographie brestoise de 1790 à 1805, dir. Ph. JARNOUX.
BESCOND, Marianne, Écriture de l'histoire et censure dans "L'Histoire de Bretagne " de Bertrand d'Argentré, dir. J. KERHERVE.

BoRDIEC, Sylvain, Aspects éducatifs et culturels du journal « Le Finistère » de 1872 à 1898, dir. M.-T. CLOITRE.

Bosser, Kristen, Étude économique et sociale de la paroisse de Pouldreuzic au XVIII siècle d'après les inventaires après décès, dir. F. RoudAuT.

CABIOCH, Élisabeth, La vie des sculpteurs grecs aux ve et IVe siècles avant JésusChrist, dir. J.-Y. ÉvEILLARD.

CADIOU, Lö̈c, La presse finistérienne face à la séparation des Eglises et de l'État, dir. M.-T. CloITRE.

CALLAREC, Laurent, Les structures sociales de Guerlesquin au XVIII siècle, dir. Ph. JARNOUX.

COUDE, Marie, Un moine fondateur au XII siècle : Étienne d'Obazine, dir. H. OUDART.

DaOUDAL, Arnaud, Les députés du Finistère à l'Assemblée législative de 1791, dir. $\mathrm{Ph}$. JARNOUX.

DOUILLARD, Éric, Un camp d'internement français : Choisel (1940-1942), dir. Ch. BOUGEARD.

Dubreuil-Le GouefFlec, Maëlle, Transcription et étude de l'inventaire de 1430 sous Jean V, duc de Bretagne, dir. J. KERHERVE.

Duval, Alan, Étude des carnets d'Anatole Le Braz de juillet à novembre 1914 : l'entrée en guerre d'un intellectuel breton, dir. M.-T. ClorTRE.

FAGES, Frédéric, La Scandinavie éclatée. De la guerre d'Hiver à la guerre du Fer, 1939-1940, dir. F. BouthILLON.

FEREC, Marie, L'impact de la guerre d'Algérie sur la vie politique à gauche dans le Finistère (1954-1962), dir. Ch. BOUGEARD.

FERNANDES, Christelle, Voleurs au bagne de Brest. 1749-1789, dir. Ph. JARNOUX.

GIRAUD, Delphine, L'utilisation du mythe dans l'idéologie impériale romaine: Alexandre le Grand ( ${ }^{e r}$ siècle av, III ${ }^{e}$ siècle après J.-C.), dir. B. LANCON.

Gonidec, Gaël, Les femmes dans les registres de la Chancellerie de Bretagne de 1462 à 1491, dir. J. KERHERVE. 
GouRIou, Nicolas, Histoire de la mémoire filmique des Américains à Brest : 19181919, dir. Y. TRANVOUEz.

GOURMELEN, Cédric, Le scrutin sénatorial dans le Finistère 1876-1914 : sa participation au processus de républicanisation, dir. M.-T. CloITRE.

Gugnalons, Anne-Laure, Les notables et l'opinion publique dans le Finistère en 1941 : comportements, attitudes et réactions, dir. Ch. BOUGEARD.

GuIRRIEC, Magali, Vivre à Landerneau au début du XVIII siècle, dir. F. Roudaut.

Hostiou, Anne, Le sport vu par " La Dépêche Sportive et Touristique " (22 novembre 1922-30 juillet 1931), dir. M.-T. CLOITRE.

JAFFRES, Pierre, La période médiévale dans la bande dessinée historico-didactique, dir. J.-C. CASSARD.

JAIN, Benoît, Les fontaines dans le pays du Porzay, dir. J.-Y. ÉvEILLARD.

JaOuen, Nathalie, Criminalité et délinquance dans la cour seigneuriale de Landerneau : 1770-1790, dir. F. RoUDAUT.

Jouan, Carole, La question laïque dans le Finistère de 1946 à 1953, dir. Ch. BOUGEARD.

Kerouedan, Yohann, Le commerce des toiles à Landerneau sous la Restauration (1815-1830) d'après les lettres de correspondance de la maison de commerce Radiguet et Goury, dir. M.-T. CLOITRE.

LASTENNET, Gwenaelle, Les évadés du Bagne de Brest d'après les registres de matricules de 1749 à 1789, dir. Ph. JARNOUX.

LE BRIS, Marie-Haud, Le règne de Jean III, duc de Bretagne (1312-1341), dir. J.-C. CASSARD.

LE DOARE, Nicolas, L'hygiène et l'embellissement de Quimper au XVIII siècle, dir. J.-L. LE CAM.

LE MeuR, Frédéric, L'orphelinat agricole et horticole de Kerbernes en Plomelin (1902-1939), dir. M.-T. CLOITRE.

LEFEBVRE, Nathalie, "In episcopatu nannetensi... ». Les chartes nantaises de Saint Florent de Saumur de 1070 à 1271, dir. J.- C. CASSARD.
LETIENNE, Ghyslain, Le baptême dans l'iconographie occidentale (II ${ }^{e}$-VII ${ }^{e}$ siècle), dir. B. LANCON.

Malleter, Laouenan Marie-Françoise, Audierne pendant la Révolution : 17891799, dir. F. RoudAuT.

MARHADOUR, Laurent, Les structures sociales de Daoulas au XVIII ${ }^{e}$ siècle, dir. Ph. JARNOUX.

Menesguen, Kristell, La municipalité Nardon : 1919-1929, dir. Y. TRANVOUEZ.

MEvel, Arnaud, Le domaine royal de Carhaix d'après le rôle-rentier de 15391542 (réformation, paysage et société), dir. J. KERHERVE.

Montesinos, Cédric, Le prix de la Rosière à Quimper de 1885 à 1945, dir. M.-T. CloITRE.

MORDIER-FLAVE, Morgane, La translation des reliques de saint Philibert et les miracles accomplis sur la route d'après Ermentaire, dir. J.- C. CASSARD.

MoRvan, Christophe, La Révolution française dans le canton de Saint-Renan (communes de Saint-Renan, Milizac, Guipronvel, Lanrivoaré, Coat-Méal et Tréouergat) : 1789-1799, dir. F. RoudAuT.

MOUllec, Sylvain, La municipalité quimpéroise au XVIII siècle, dir. J.-L. LE CAM. NADAN, Gaëlle, L'évolution des prénoms dans les paroisses du nord du Pays bigouden (1702-1822), s.l., s.n., 1999, 156 p., dir. J.- L. LE CAM.

NICOLAS, Iwan, La guerre d'Espagne à travers le témoignage d'un socialiste libertaire français : Gaston Leval, 1895-1978, dir. Y. TRanvouez.

OLLIVIER, Cécile, Le Transfert des cimetières dans les cantons de Douarnenez, PontCroix, Plogastel-Saint-Germain, PontL'Abbé, et Quimper au XIXe siècle et au début du XX $x^{e}$ siècle, dir. M.-T. CLOITRE.

ORLAC'H, Laurence, Les femmes détenues à la prison de Brest (1831-1851), dir. J.Y. CARLUER.

PARROT, Ingrid, Transcription et étude $d u$ registre $B 11$ des lettres scellées à la Chancellerie de Bretagne en 1487-1488, dir. J. KERHERVE.

PENSART, Frédéric, Le Japon vu par « La Dépêche de Brest et de l'Ouest » (19301941), dir. Ch. BOUGEARD. 
PREGat, Anne, Le culte du soleil à Rome et l'idéologie du pouvoir impérial (III IV siècle après J.-C.), dir. B. LANCON.

QUENTEL, Julien, L'aventure politique de Georges Bernanos, dir. Ph. BRunET.

Quiniou, Caroline, Le Père Yvon de Guengat. Portrait d'un catholique social, dir. Y. TRanvouez.

QuINTIN, Virginie, Élus municipaux et cantonaux de l'arrondissement de Brest (1934-1951), dir. Ch. BOUGEARD.

RAER, Katell, La vie religieuse dans le canton de Carhaix du Concordat à la laïcisation, dir. M.-T. CLOITRE.

RAguenez, Yvan, La scission CGT-FO dans le Finistère, dir. Ch. BOUGEARD.

RALON, Gilles, Commerce maritime et négociants à Paimpol et Bréhat : 1750-1793, dir. Ph. JARNOUX.

RAME, Paul, La police dans le Finistère pendant l'Occupation et à la Libération. Étatisation, Collaboration, Épuration. La police finistérienne face à la guerre (1940-1950), dir. Ch. BOUGEARD.

RAPHALEN, Sylvia, La réclame dans deux journaux brestois, "l'Océan " et "l'Armoricain" sous le Second Empire, dir. M.-T. CloITRE.

RIDENT, Marie-Anne, La chambre de commerce de Brest : acteur de la vie économique brestoise (1919-1938), dir. Y. TRANVOUEZ.

RoUDAUT, Anne-Laure, Le gouvernement belge de Londres, De Gaulle et la France libre : 1940-1944. La souveraineté de la Belgique au travers des relations belgogaullistes, dir. Ch. BOUGEARD.

ROUQUETTE-DESBORDES, Geneviève, Les lois scolaires sous Vichy et leur application dans le Finistère, dir. Ch. BOUGEARD.

SABATIER, Benjamin, Les reconstructions d'églises paroissiales à Concarneau aux XIX et XX $X^{e}$ siècles (1802-1997), dir. M.T. CloITRE.

SADAOUI, Rachid, Le lycée de La Tour d'Auvergne (1930-1960) ou l'impact de la Seconde Guerre mondiale dans l'histoire de l'établissement, dir. M.-T. CLOITRE.

SCOUARNEC, Morgane, Les manoirs des $X V^{e}$ et $X V I^{e}$ du canton de Plouigneau, dir. J. KERHERVE.
TALLEC, Ronan, Le Kavalierstour dans les biographies de sermons funèbres : un marqueur social des élites protestantes allemandes au XVI ${ }^{e}$ siècle, dir. J.- L. LE CAM.

TiRILLY, Lionel, Étude sociale des gens de justice de la baronnie du Pont au XVIII siècle (1700-1780), dir. F. RoudAuT.

TREGOAT, Mickaël, Guerre froide et Marvel Comics, dir. Ph. BRUNET.

TREMOUREUX, Jean-François, L'abbaye NotreDame de Timadeuc de 1841 à 1922, dir. Y. TRANVOUEZ.

Youinou, Frédéric, Genèse d'une intégration: les Barbares dans les hautes sphères civiles de l'Occident romain de Trajan à Valentinien II, dir. B. LANCON.

\section{8}

BECAM, Marc, La Dépêche de Brest et la montée du nazisme (1933-1939), dir. Ch. BOUGEARD.

Boudigou, Sophie, La presse et la sortie de la France de l'OTAN, dir. F. BOUTHILLON.

BOUYER, Stéphanie, Les Joly de Rosgrand : une famille de la bourgeoisie quimperloise au XVIII siècle, dir. Ph. JARNOUX.

BRELIVET, Alain, La culture de l'esprit d'apostolat à l'Institution Notre-Dame du Kreisker et à l'Institution Saint-François : une approche de la formation chrétienne dans les grands collèges catholiques $d u$ Léon des années vingt aux années quarante, dir. Y. TRANVOUEZ.

CARN, Laurent, Du fait à la cause : représentations des prêtres-ouvriers dans la presse catholique 1955-1965, dir. Y. TRANVOUEZ.

Chevalereau, Fabienne, Les cités de la Pamphilye romaine à l'époque impériale, dir. B. LANCON.

Claquin, Loïc, L'historien et la Cinémathèque de Bretagne : approches de la triple fonction de l'historien : aspects de la recherche juridique, aspects de la recherche historique, déontologie dans l'utilisation des images d'archives, dir. Y. TRANVOUEZ.

Corc, Isabelle, Les délits maritimes et fluviaux dans l'arrondissement de Quimper 1811-1896, dir. J.-Y. CARLUER.

$\mathrm{Coz}$, Christelle, Transcription et étude du registre des lettres scellées à la 
Chancellerie de Bretagne en 1509, dir. J. KERHERVE.

DANAN, Paul-Simon, Les relations diplomatiques du Portugal pendant la Guerre d'Espagne (1936-1939), dir. F. BouTHILLON.

DeSHOUX, Esther, La passion de saint Julien et les débuts de son culte, dir. H. OUDARD.

DESROCHES, Élisabeth, Introduction à l'étude de " la Semaine religieuse » du diocèse de Quimper et de Léon (1886-1912), dir. M.-T. CLOITRE.

FERREC, Solenn, « La peinture bat son plein et son charme... "Le-Faouët et les artistes des années 1845 à la fin des années 1930, dir. M.-T. CloITRE.

Gullou, Stéphanie, Transcription et étude du registre des lettres scellées à la Chancellerie de Bretagne en 1520, dir. J. KERHERVE.

HALET, Marie, Le renouveau théâtral à Brest après la Seconde Guerre mondiale, dir. M.-T. CLOITRE.

HALl, Christelle, Les propagandes, les médias et l'opinion publique dans le Finistère, en 1942, dir. Ch. BOUGEARD.

HASCOET, Nathalie, Fêtes et spectacles dans l'agglomération brestoise sous l'Occupation (1940-1944), dir. Y. TRANVOUEZ.

HEREMBERT, Frédéric, La papauté et l'abbaye Sainte-Croix de Quimperlé (XI'-XVe siècles), dir. J. KERHERVE.

JANNIC, Magali, Morgat 1880 : la naissance d'une plage, dir. M.-T. CLOITRE.

JEgou-Cochard, Christelle, Charles Cornic (1731-1809) "l'officier bleu": la plus belle illustration maritime de Morlaix au XVIII siècle, dir. F. RoudAuT.

KahouAJ, Samir, Les visites des "Imperatores "romains en Égypte, de Jules César à Dioclétien, dir. B. LANCON.

KeRBIQUET, Vincent, De l'exemple à l'exil : le rôle de l'Irlande dans le mouvement nationaliste breton, dir. Ch. BOUGEARD.

KERMOAL, Emmanuelle, Le collège NotreDame du Kreisker de Saint-Pol-de-Léon 1945-1983, dir. Y. TRANVOuEZ.

Lagadec, Claire, Transcription et étude d'un inventaire d'archives de Bretagne $X I V^{e}-X V^{e}$ siècles, dir. J. KERHERVE.
LE Bot, Anne, Les thermes gallo-romains en Armorique, dir. J.-Y. ÉvEILLARD.

LE BRETON, Arnaud, L'évolution du communisme rural dans le sud-ouest des Côtes-du-Nord 1958-1983 (cantons de Callac, Gouarec, Maël-Carhaix, Rostrenen et Saint-Nicolas-du-Pélem), dir. Ch. BOUGEARD.

LE GoUIC, Laurence, Les romains et l'art grec au $1^{\text {er }}$ siècle avant Jésus-Christ à travers les arts plastiques, dir. M.-Cl. L'HULLLER.

LE GoulL, Sandrine, L'agriculture en Léon et Cornouaille au XVIII siècle, dir. Ph. JARNOUX.

LE GRAND, Morgane, Brest au XVIII siècle, ou l'émergence d'une bourgeoisie négociante, dir. Ph. JARNOUX.

LE Loc'H, Frédéric, Sébastien Jartel (17981878) : un curé du XIXe siècle, dir. M.-T. CloItre.

LE LOUET, Bran, Le bagne et l'exclusion : mendiants et vagabonds au bagne de Brest, dir. Ph. JARNOUX.

LE Mouellic, Sandrine, Les révoltes égyptiennes du fr $^{\text {au III }}$ siècle après J.-C., dir. B. LANCON.

LE PENNEC, Christophe, Recherches sur les origines antiques de Vannes (Darioritum), dir. J.-Y. ÉvEILLARD.

LE Pichon, Laurent, L'abbaye de SainteCroix de Quimperlé au XVIII siècle : propriétés, revenus et vie économique (1717-1790), dir. Ph. JARNOUX.

LE Roux, Lionel, Deux paroisses et la mer: Porspoder et Landunvez au XVIII siècle, dir. Ph. JARNOUX.

LETELLIER, Laure, L'alphabétisation à Plougastel au XVIII siècle, dir. J.- L. LE CAM. LETOURNEUR, Marie-Joëlle, L'esclavage et la dépendance dans la correspondance de saint Jérome, dir. M.-Cl. L'HUILLIER.

LOARER, Agnès, Le traditionalisme à la base : les lettres de la Chouanne (Morlaix 1970-1982), dir. Y. TRANVOUEZ.

LOUARN, Philippe, La remise en cause des droits féodaux dans le Finistère. 4 août 1789-17 juillet 1793, dir. Ph. JARNOUX.

MARHADOUR, Christelle, Le Bureau de bienfaisance de Brest de 1870 à 1914, dir. M.-T. CLOITRE.

MARLIER, Jean-Noël, La correspondance du Préfet maritime de Brest - ordres et 
décisions - pendant la campagne du Mexique, dir. J.-Y. CARLUER.

MARTIN, Emmanuel, Les Prêtres-Ouvriers à travers “Courrier PO »(1971-1995), dir. Y. TRANVOUEZ.

MARZIN, Aurore, La découverte de l'Indochine (1860-1893) dans la "Revue maritime et coloniale », dir. M.-T. CLOITRE.

MERRIEn, Nicolas, Données sur l'effort de guerre à Brest 1914-1918, dir. M.-T. Cloitre.

MEVEL, Agnès, Violences sexuelles à l'encontre des femmes et des enfants au XIX siècle dans le Finistère, dir. J.-Y. CARLUER.

Minıou, Éric, Corentin le-Floch. Député paysan aux États généraux de 1789. Biographie "impressionniste " d'un laboureur bas-breton (1754-1794), dir. $\mathrm{Ph}$. JARNOUX.

MoAl, Sophie, Les conscrits du NordFinistère d'après les registres matricules de 1870, dir. J.-Y. CARLUER.

Moysan, Lise, Délinquance, criminalité et répression : la jeunesse déviante dans l'arrondissement de Quimper (1847-1896), dir. J.-Y. CARLUER.

PHILIP, Armelle, René Barjavel (1911-1985). Itinéraire d'un antimoderniste, du pétainisme à l'écologisme, dir. F. BOuTHILLON.

PRIOUx, Élodie, Gaston Chabal (1882-1965) : itinéraire d'un architecte du XX siècle à la personnalité singulière, dir. M.-T. Cloitre.

QUERREC, LOÏc, Être meunier dans la région quimpéroise au XVIII siècle : Bodivit, Briec, Combrit, Cuzon, Ergué-Armel, Ergué-Gabéric, Kerfeunteun, Pluguffan, Quimper, Saint-Evarzec, dir. J.- L. LE CAM.

RENEZ, Emmanuelle, L'activité maritime et commerciale des Provinces-Unies sur les côtes du Finistère 1690-1790, dir. Ph. JARNOUX.

RICHARD, Sophie, Les instituteurs publics et la vie scolaire dans le Finistère de 1939 à 1945, dir. Ch. BOUGEARD.

RIVIERE, Christophe, Notables municipaux et élus départementaux en Morbihan (1932-1959), dir. Ch. BOUGEARD.

RONARC'H, Marine, Les missions d'OutreMer dans "la Semaine religieuse de Quimper et de Léon » (1886-1962), dir. M.-T. CLOITRE.
Ropars, Hélène, L'enquête Boulard dans le diocèse de Quimper et de Léon (19571958), dir. Y. TRANVOUEz.

SQUIVIDAN, Isabelle, La presqu île de Crozon sous l'Occupation : la vie quotidienne des presqu'îliens de juin 1940 à septembre 1944, dir. Ch. BOUGEARD.

Stefanelly, David, Lettres d'un député : Paul Dieuleveult (1799-1867) représentant des Côtes-du-Nord à l'Assemblée Législative (1849-1851), dir. J.-Y. CARLUER.

STEPHANY, Aurélien, Recherche des itinéraires antiques dans le triangle Carhaix, Lannion, Morlaix, dir. J.-Y. ÉVEILLARD.

THERSIQUEL, Loïc, Le théâtre de Quimper (1904-1998). Histoire d'un théâtre municipal de province, dir. M.-T. CLOITRE.

VEILLARD, Emmanuel, Étude du cartulaire de l'abbaye Saint-Sulpice de Bourges (1064-1137), dir. H. OUDART.

VIROT, Emmanuel, Les reconstructions d'églises paroissiales dans le Léon au xixème siècle (1814-1905), dir. M.-T. CloITRE.

Voquer, Martine, Histoire de la Caisse régionale de Crédit Agricole du Finistère 1907-1950, dir. Ch. BOUGEARD.

\section{7}

Balbous, Olivier, La chambre de commerce de Brest, acteur du développement brestois (1898-1920), dir. Y. TRANVOUEZ.

BERNARD, Norbert, La seigneurie des rives de l'Odet (1425-1575), dir. J. KERHERVE.

BOURDONNAY, Fanny, Être bourgeois en Bretagne intérieure XVII ${ }^{\mathrm{e}}$-xIX ${ }^{\mathrm{e}}$ siècles : la famille Allain (de) Launay, dir. J.Y. CARLUER.

BOURVEn, Virginie, Étude démographique de la paroisse de Saint-Mathieu (Morlaix) au XVII' siècle, dir. F. RoudAuT.

CASTILLE, José, Une famille cornouaillaise, du début du XVII siècle à la Révolution (1607-1789) : les Roignant, dir. F. RoUDAUT.

Chevassus, Ludovic, La guerre chez les écrivains de l'Empire Romain de la fin $d u e^{e r}$ siècle à la fin du Ive siècle. Évolution de l'idéologie et de la représentation, dir. M.-Cl. L'HUILLIER.

Christien, Karine, Démographie, alphabétisation et société à Quimperlé : 17501788, dir. J.-L. LE CAM. 
CloITRE, Anne-Bénédicte, L'imprimerie dans le Finistère au temps de la réglementation : 1810-1870, dir. J.-Y. CARLUER.

Collin, Philippe et RaOul, Philippe, L'Épuration dans le Finistère : 1944/1946. Des soupçons à la peine de mort. La répression de la Collaboration sur le plan administratif et judiciaire dans un département breton, dir. Ch. BOUGEARD.

CONAN, Hélène, La transition des pouvoirs à la Libération dans l'arrondissement de Quimper (août 1944-septembre 1945), dir. Ch. BougEard.

Cousin, Thomas, L'athlétisme dans la Grèce antique, de la période archä̈que à la période héllénistique, dir. J.-Y. ÉVEILLARD.

DANIEL, Florence, Quelques approches de l'image de la femme à travers la presse catholique de l'entre-deux-guerres (19191939) dans le Finistère, dir. Y. TranVOUEz.

DEBORD, Karine, Étude et transcription du registre des lettres scellées à la Chancellerie de Bretagne en 1510, dir. J. KERHERVE.

DIAMANT, Marie-Gwenn, L'enseignement primaire à Landerneau des années 1820 à la veille des lois Ferry, dir. M.-T. CLOITRE.

EOzENOU, Isabelle, Les négociants bretons à Cadix (1778-1808), dir. Ph. JARNOUX.

FEREC, Yann, Morvan Lebesque au Canard Enchaîné. Une lente et tortueuse pérégrination vers Comment peut-on être Breton?, dir. Y. TRANVOUEZ.

FERREYRolle, Catherine, Transcription et étude d'un ensemble d'archives privées d'origine léonarde (XIVe-XVIe siècles), dir. J. KERHERVE.

FORLODOU, Guillaume, L'enfance abandonnée à Quimper de 1661 à 1791, dir. J.-L. LE CAM.

GentiL, Mathieu, Étude quantitative de délits et de délinquants dans l'arrondissement de Quimper (1936-1946), dir. J.-Y. CARLUER.

GoasdufF, Patrice, Étude du film Départs, 1953, dir. Y. TranvouEz.

Gratesac, Tristan, L'expédition d'Irlande de 1796, dir. Ph. JARnOUX.

GuEdEs, Jean-Marie, Les événements d'Algérie à travers le quotidien OuestFrance (novembre 1954-octobre 1956), dir. Ch. BOugEard.
GuILlEmin, Joseph, En Arrée et Poher : un monde rural au XVIf siècle. Une paroisse de Cornouaille : Scrignac en pays de Poher de Louis XIII à Louis XV, dir. Ph. JARNOUX.

Gulllerm, Paul, Images du totalitarisme en bande dessinée, dir. F. BouTHILLON.

HENRY, Jacqueline, Les dix premières années du Bagne de Brest : 1749-1759, dir. Ph. JARNOUX.

HiRrIEN, Hélène, Un syndicat pour les ouvriers agricoles : du Syndicat des ouvriers agricoles d'Elliant à la Fédération générale agro-alimentaire de la CFDT (1936-1990). Évolution du salariat agricole dans le Finistère de l'entre-deuxguerres à nos jours, dir. M.-T. CLOITRE.

Hostıou, Franck, Le RPF dans le Finistère, dir. Ch. BOUGEARD.

HuTH, Olivier, Étude d'un journal républicain Le Réveil du Finistère (1904-1909), dir. M.-T. CLOITRE.

JACQ, Marie-Aude, Le mouvement Poujade dans le Finistère de 1955 à 1971, dir. Y. TranvouEz.

KERMARREC, Juliette, Transcription et étude du registre des lettres scellées à la Chancellerie de Bretagne en 1508, dir. J. KERHERVE.

LE BoulANGER, Anne-Laure, La question de la main-d'œuvre dans le Finistère. 1940-1944, dir. Ch. BOUGEARD.

LE BRAS, Éric, L'opinion publique finistérienne face au cartel des gauches : 19241926, dir. Y. TRANVOUEz.

LE DERofF, Catherine, La vie quotidienne des fortes têtes du Bagne de Brest (d'après les registres de matricules de 1749 à 1765), dir. Ph. JARNOUX.

LE FLOCH, Gilles, Les capitaines de navires dans l'Amirauté de Cornouaille de 1755 à 1789, dir. Ph. JARNOUX.

LE GALL, Myriam, Les manoirs de la presqu'île saint-politaine aux $X V^{e}$ et $X V I^{e}$ siècles. (Communes de l'île de Batz, Plouénan, Plougoulm, Roscoff, SaintPol-de-Léon, Santec, Sibiril), dir. J. KERHERVE.

LE GALL, Vanina, Les luttes sociales à Brest (mai 1947-avril 1950), dir. Y. TranVOUEZ. 
LE Goc, Sophie, Le canton de Quimperlé dans les années noires 1939-1945, dir. Ch. BOUGEARD.

LE Goff, Émmanuelle, Les débuts de La Résistance. Journal royaliste et catholique de Morlaix (1885-1893), dir. M.-T. CloItre.

LE GoFf, Stéphanie, Quimper : un chef-lieu départemental dans la guerre (novembre 1938-décembre 1941), dir. Ch. BOUGEARD.

LE GRAND, Fanny, Coups et blessures dans l'arrondissement de Quimper au XIXème siècle (1811-1868), dir. J.-Y. CARLUER.

LE Guen, Arnaud, Le canton de Châteauneuf-du-Faou sous la Révolution (1789-1799), dir. F. RoudAuT.

Le Guen, Patrice, L'alphabétisation à Ploudalmézeau de 1700 à 1792, 1997, 137 p.; dir. J.-L. LE CAM.

Le PeRsonnic, Frédéric, L'abbé Louis LeClerc « Kloareg ar Wern » (1861-1944) ou la politique du breton d'un abbé régionaliste, dir. M.-T. CLOITRE.

LE SEAC'H, Emmanuelle, Les crossettes et les gargouilles dans quatre cantons $d u$ Finistère : Landerneau, Landivisiau, Ploudiry, Sizun, dir. J.-Y. ÉVEILLARD.

LESSARD, Sophie, Les reconstructions d'églises dans la Cornouaille finistérienne au XVIII siècle, dir. J. TANGUY.

MALO, Christine, La population hospitalière de Morlaix (d'après les registres de décès de l'hospice civil de Morlaix) : 1813-1863, dir. M.-T. CloITRE.

MARC, Nicolas, Étude des dons et legs à Quimper au XIXe siècle (1809-1914), dir. M.-T. CLOITRE.

MARECHAL, Sophie, La criminalité dans la sénéchaussée royale de Carhaix : 17701790, dir. Ph. JARNOUX.

MARTIN, Yves, Les fontaines du canton de Ploudalmézeau, dir. J.-Y. ÉvEILLARD.

NOURRY, Audrey, Les manoirs des XV et XVI siècles au cœur du Léon (communes de Bodilis, Cléder, Lanhouarneau, Mespaul, Plouescat, Plougar, Plounévez-Lochrist, Plouvorn, Plouzévédé, Saint-Vougay, Tréflaouénan, Tréflez, Trézilidé), dir. J. KERHERVE.
Pensart, Claude, Société et alphabétisation à Audierne au XVIII ${ }^{e}$ siècle, dir. J.-L. LE CAM.

Plouzane, Yves-Marie, La moyenne délinquance dans l'arrondissement de Châteaulin (1835-1859), dir. J.-Y. CARLUER.

PoQuet, Sébastien, Les élus du Finistère et la Guerre d'Algérie (1958-1962), dir. Ch. BOUGEARD.

Queguineur, Frédéric, Le collège SaintFrançois de Lesneven : 1945-1975, dir. Y. TRANVOUEZ.

QUENEHERVE, David, Une famille de cultivateurs cornouaillais au dix-neuvième siècle : les Sinquin, dir. M.-T. CloITRE.

REMEUR, Hélène, Étude des costumes dans les grands calvaires du Finistère, dir. J.-Y. ÉVEILLARD.

SAVINA, Marc, Louis XVI : deux cents ans d'historiographie française, dir. F. BOUTHILLON.

SERGENT, Cyrille, La défense des côtes $d u$ Léon durant la Révolution française (1789-1799), dir. Ph. JARNOUX.

Simon, Marie, Vie économique et sociale à Logonna-Daoulas de 1866 à 1914, dir. J.-Y. CARLUER.

Stephan, Murielle, Corsaires brestois et vent d'Amérique : 1778-1783, dir. Ph. JARNOUX.

TheBAult, Philippe, La vie quotidienne des habitants du canton de Jugon pendant la Seconde Guerre mondiale (19391945), dir. Ch. BOUGEARD.

Thuault, Ludovic, Les comptes de la seigneurie de Goëlo : 1480-1491, dir. J. KERHERVE.

YvEn, Estelle, La préhistoire et la protohistoire sur le canton de Lanmeur, dir. J.-Y. ÉVEILLARD.

\section{6}

AUFFRET, Yann, Gilles de Rais, de l'histoire au mythe, dir. J. KERHERVE.

Bargain, Frédéric, Auguste Dupouy et l'" l'Histoire de la Bretagne ", dir. J. KERHERVE.

BESSELIEVRE, Jean-Yves, Les travaux de fortification de Brest à la fin du XVIII siècle (1776-1784.), dir. F. RoudAuT.

BLAISE, Yann, L'inventaire des statues géminées du Nord-Finistère, dir. J.-Y. ÉVEELLARD. 
BodET, Stéphanie, La Révolution dans les communes de Querrien, Saint-Thurien et Scaër 1789-1799, dir. F. RoudAuT.

BRAMOULlE, Mickaël, Lannilis : un canton du Finistère dans la tourmente 19391945, dir. Ch. BOugEARD.

CAMPION, Armelle, Les artistes de Quimper aux XVII et XVIII siècles, dir. J.-L. LE CAM.

CARO, Laurent, Le Finistère et Maurice Bouilloux-Lafont député de Quimper (1914-1932), ligne politique (1914-1939), dir. M.-T. CLOITRE.

Cenac, Juliette, La guerre d'Indochine au travers de la presse bretonne, dir. Ch. BOUGEARD.

CHAURIS, Anne, Le poids financier des enclos paroissiaux léonards, dir. F. ROUDAUT.

CLEC'H, Gaëlle, La vie municipale à Morlaix à l'époque du Second Empire (18521870), dir. M.-T. CloITRE.

Colin, Dominique, Les comptes de la cathédrale de Quimper de 1439 à 1514, dir. J. KERHERVE.

DANGUY-DES-DESERTS, Marie, Transcription et étude du registre $B 14$ des lettres scellées à la Chancellerie de Bretagne en 1503, dir. J. KERHERVE.

Davis, Anne et Memain-Bodilis, Muriel, Étude démographique de la ville de Landerneau 1670-1790, dir. J.-L. LE CAM.

FAUTREL, Cédric, La civilisation assyrienne et la France du XIX siècle. De la découverte à la muséologie, dir. $\mathrm{M}$.-Cl. L'HUILLIER.

Galic, Bertrand, Armand Rousseau, gouverneur général de l'Indochine française (27 décembre 1894-10 décembre 1896), dir. M.-T. CloITRE.

GRATAS, Ludivine, Entre Plantagenêts et Capétiens, la Bretagne face à ses choix dans " l'Histoire »de Bertrand d'Argentré, dir. J. KERHERVE.

GRATON, Emmanuel, Les enceintes urbaines et militaires gallo-romaines dans la péninsule armoricaine, dir. J.Y. ÉVEILLARD.

GREE, Karine, Les capitaines de navires dans l'Amirauté de Cornouaille au XVIII siècle, dir. Ph. JARNOUX.

Gullet, Élisabeth, " Les chroniques des rois, ducs et princes roiaulx de la
Bretaigne armoricane aultrement nommée la moindre Bretaigne ", de Pierre Le Baud, dir. J.-C. CASSARD.

GullLouAIS, Anne-Laure, Transcription et étude du registre $B 15$ des lettres scellées à la Chancellerie de Bretagne en 1505, dir. J. KERHERVE.

Hamon, Nathalie, Saint Vincent Ferrier, la Bretagne et les Bretons, dir. J.-C. CASSARD.

KeRSCAVEN-DoucEn, Gaëlle, Les archives anciennes de la ville de Quimper, dir. J. KERHERVE.

LAHELLEC, Philippe, Approche de la vie et de l'oeuvre du fondateur de l'hagiographie bretonne: Albert Le-Grand de Morlaix et "La vie des Saincts de la Bretaigne Armorique (1637) ", dir. B. TANGUY.

LASBLEYE, Erwan, La santé à bord des navires de la Marine d'après les "Archives de médecine navale de 1864 à 1900, dir. M.-T. CLOITRE.

LAURIER, Laurent, Notre-Dame de Kerbonne : origines et évolution d'une paroisse de la rive droite de Brest (18921958), dir. Y. TRANVOUEZ.

LEDOIT, Sandrine, Fatalité, destin et libre arbitre des héros, dir. J.-Y. ÉVEILLARD.

LE CORRE, Martine, Quelques aspects de la vie quotidienne à Lannion pendant la Seconde Guerre mondiale (19391945), dir. Ch. BOUGEARD.

LE GALL, Edith, Le fonctionnement de la seigneurie de Kerouzere 1680-1789, dir. J. TANGUY.

LE Goulc, Patrick, La conscription dans l'arrondissement de Châteaulin (18071810), dir. J-Y. CARLUER.

Le MaleFan, Serj, Callac : une cité rurale au XIXe siècle, dir. M.-T. CLOITRE.

LE POULEuF, Frédéric, Origine et naissance d'une commune : Le-Relecq-Kerhuon (1863-1903), dir. M.-T. CLOITRE.

LE Pouleuf, Nathalie, Les organes de presse d'Henri Ansquer : des "Commérages brestois » au "Penn-Baz»1914-1919: le Pen-Baz: un journal satirique et littéraire pendant la Grande Guerre, dir. F. BOUTHILLON.

LE REST, Stéphane, Les chantiers paroissiaux en Cornouaille finistérienne $d u$ $X V I^{e}$ au XVIII siècle, dir. F. RoUDAUT. 
Le Roux, Bruno, Le mouvement Croix de Feu et le PSF dans le Finistère, dir. F. BOUTHILLON.

L'HeLGUEN, Caroline, Les reconstructions d'églises dans le Léon au XVIII siècle, dir. F. RoudAut.

LOUEDEC, Patricia, Paysage urbain et propriétaires carhaisiens sous le règne de Louis XIV, dir. Ph. JARNOUX.

MeLLOUET, Pierrick, Les activités agricoles dans le pays pagan au cours de l'entredeux-guerres (Plounéour-Trez et Brignogan), dir. M.-T. CloITRE.

MiLIN, Rozenn, Les Celtes et la mort dans l'Antiquité d'après les textes grecs et latins, dir. J.-Y. ÉVELLLARD.

MOAL, Laurence, Bertrand d'Argentré. Patriote ou historien?, dir. J. KERHERVE.

NICOLAs, François, Corps et société. Le discours du corps au Ive siècle de notre ère, dir. M.-Cl. L'HUILLIER.

OLLIVIER, Marc, L'histoire médiévale dans le «Bulletin de la société archéologique du Finistère », dir. J. KeRHERvE.

PAILLER, Yvan, Inventaire \& prospections archéologiques dans le canton de Ploudalmézeau, dir. J.-Y. ÉVEILLARD.

PARC, Isabelle, Les manoirs du pays des Abers aux XVe et XVI siècles (communes de Guissény, Landeda, Lannilis, Plouguerneau, Saint-Frégant, dir. J. KERHERVE.

PAUBERT, Laurent, La vie politique en Pays Bigouden entre les 2 guerres (19191940), dir. M.-T. CloITRE.

PetrT, Caroline, Le port de Quimper de 1840 à 1939, dir. M.-T. CLOITRE.

PICQUET, Alan, Landerneau 1939-1945, dir. Ch. BOUgeard.

Postec, Isabelle, La vie quotidienne dans le canton de Lesneven pendant la guerre 1939-1945, dir. Ch. BOUGEARD.

Postic, Yann, L'occupation allemande à Brest de 1940 à 1942 : les rapports occupants-occupés ou l'impact des opérations militaires sur l'opinion publique, dir. Ch. BOUGEARD.

QUEINNEC, Jacques, Les comptes de la prévôté de Paris dans la $1^{r e}$ moitié su XIII siècle, dir. J. KERHERVE.

Rault, Carl, La chronologie de la Guerre de Succession de Bretagne, dir. J.C. CASSARD.
REungOAT, Magali, Le Trésor de l'Espagne des ducs de Bretagne, d'après le compte de Guillaume Chauvin (1469-1472), dir. J. KERHERVE.

ROGER, Éric, Société et vie quotidienne à Brest de 1728 à 1742 d'après les inventaires après décès, dir. F. RoudauT.

Rosec, Florence, Les artistes brestois de 1670 à 1789, dir. F. RoudAut.

RoudAuT-ADAm, Valérie, Réédition des cartulaires de l'église cathédrale SaintCorentin de Quimper, dir. J. KERHERVE.

Roussin, Isabelle, Les biens fonciers de l'abbaye du Relec aux XVIT et XVIII siècles, dir. Ph. JARNOUX.

SAlaun, Loïc, La délinquance dans un arrondissement finistérien : l'exemple de Quimperlé (1811-1852), dir. J.Y. CARLUER.

Stroobants, Virginie, Bertrand d'Argentré et l' Histoire de Bretagne ", dir. J. KERHERVE.

TRIVIDIC, Erwan, Analyse et perception d'un évènement en temps de guerre: la Révolution russe vue par "La Dépêche de Brest », dir. F. BouthILLON.

Trividic, Stéphanie, Les domestiques à Brest au XVIIIème siècle, 1996, 130 p.; dir.?

VILATTE, Ludovic, La vie politique et syndicale dans l'arrondissement de Quimper en 1947, dir. Ch. BOUGEARD.

STEREN, Anne, Les congrégations religieuses féminines de Quimper, dir. M.-T. CLOITRE.

\section{5}

Ambroise-Rendu, Guillaume, Gales, Gwenaële et LE BRAS, Valérie, Inventaire des archives du château de Keruzoret (Plouvorn), dir. J. TANGuY.

Arzur-Treguier, Anne-Marie, Monsieur l'abbé Jacques Calvarin et le Kannadik Treglonou, dir. Y. TRANVOUEZ.

BERRIVIN, Antoine, Pèlerinages et politique. L'exemple de la dévotion à Notre-Dame du Folgoët, 1888-1954, dir. Y. TRANvouez.

BiLly, Cyril, Les voyages français autour du monde vus à travers les "Annales maritimes et coloniales »1817-1841, dir. M.-T. CLOITRE. 
BouCHARD, Karine, Femmes, images de la femmes et représentation chez saintJérôme, dir. M.-Cl. L'HuILLIER.

BUREL, Denis, Ballades et chansons politiques de la rébellion irlandaise de 1798, dir. F. RoudAuT.

CARAES, Laurent, Création artistique et aménagement de l'église de Saint-Jean-duDoigt aux XVI et XVIT siècles, dir. J. TANGUY.

Chardon, Laurent, Criminalité et société de 1760 à 1789 d'après le fonds du Présidial de Quimper, dir. F. RoudAuT.

DERRIEN, Maryvonne, Une commune bretonne sous l'occupation allemande: Brennilis, dir. F. BouTHILLON.

Ely, Marie-Gabrielle, Les Français prisonniers sur parole en Grande-Bretagne de 1756 à 1815, dir. J. TANGUY.

EOZENOU, Éric, La vie quotidienne à Lannilis au XVIII siècle, d'après les inventaires après décès, dir. F. RoudAuT.

FACCENDA, Sébastien, Société et vie quotidienne à Brest de 1776 à 1789 d'après les inventaires après décès, dir. F. ROUDAUT.

FLOCH, Arnaud, L'occupation allemande dans le Finistère en 1942, dir. M.-T. CLOITRE.

FLOCH, Sandrine, Le patronage religieux des ducs Jean IV et Jean V dans le duché de Bretagne, dir. J.-C. CASSARD.

GASPARUTTO, Claire, Guerre et religion pendant la Grande Guerre à travers "Le Courrier du Finistère », dir. Y. TRAnVOUEz.

GAUTIER, Élisabeth, "L'Impartial $d u$ Finistère ", journal catholique et légitimiste de combat au début de la III République (1870-1883), dir. M.-T. CloITRE.

Godoc, Patrice, Nicias, dir. J.-Y. ÉvEILLARD.

Grimal, Constance, Le Trésor de SaintUniac, dir. J.-Y. ÉVEILLARD.

Gueguen, Hervé, Le canton de Scrignac pendant la Révolution (1789-1799), dir. F. ROUDAUT.

GuENOLE, Ghislaine, Une famille bretonne au bas Moyen Âge : les Du-Chastel XIV $X v^{e}$ siècles, dir. J. KERHERVE.

Guillou, Loïc, Le collège jésuite de Quimper (1620-1762), dir. J.-L. LE CAM.
GUYOMAR, Gwénaëlle, Monographie de l'abbaye Saint-Maurice de Carnoët, dir. J. TANGUY.

KERDONCUFF, Didier, Moulins et meuniers dans la région d'Irvillac au XVII' siècle (Irvillac, Daoulas, Dirinon, Hanvec, Hôpital-Camfrout, Logonna, Rumengol, Saint-Eloy, Saint-Urbain, Trévarn), dir. J. TANGUY.

KERMOAL, Benoît, La carrière politique de Jean-Louis Rolland de 1919 à 1946, dir. M.-T. Cloitre.

Keromes, Françoise-Anne, Peut-on être historien et breton dans la France royale ou «l'Histoire de Bretagne ». Transcription et comparaisons commentées de 1583, 1588 et 1618 , dir. J. KERHERVE.

LAGADEC, Yves-Hervé, Les fontaines $d u$ canton de Plabennec, dir. J.-Y. ÉVEILLARD.

LEBDIRI, Sébastien, Études mégariennes, dir. J.-Y. ÉVEILLARD.

LE Borgne, Roland, Société et vie quotidienne à Brest à la fin du XVIf et au début du XVIII siècle d'après les inventaires après décès, dir. F. RoudAuT.

LE CALVE, Jacqueline, Kernault, la mémoire d'un lieu : de l'évolution du rôle $d u$ manoir dans le milieu rural $X v^{e}-X X^{e}$ siècle, dir. J. KERHERVE.

LE GALL, Bruno, La Révolution dans le canton de Pont-Croix (1789-1799), dir. F. ROUDAUT.

LE SECH, Anaig, Étude démographique des paroisses de Lanmeur et de Plouézoc'h à la fin du XVII siècle et au XVIII siècle, dir. J. TANGUY.

LE VAllLANT, Michel, La seigneurie de Maillé en Bretagne 1577-1747, dir. J. KERHERVE.

LEOST, Pierre, La présence française sur le French-Shore (1713-1904) : aspects diplomatiques et institutionnels (d'après les archives de la division navale de Terre-Neuve), dir. J.-Y. CARLUER.

LIGEN, Joëlle, Les étrangers dans l'arrondissement de Brest, de 1815 à 1875, dir. J.-Y. CARLUER.

MAHE, Sylvie, Les femmes et l'ascétisme d'après la correspondance de saintJérôme (milieu du I $v^{e}$-début du $v^{e}$ siècle), dir. M.-Cl. L'HUILLIER. 
MENEC, Sandrine, «L'embellissement » de la ville de Brest au XVIII siècle, dir. J.L. LE CAM.

Michel, Anne, Yves Michel, architecte d'art sacré 1910-1970, dir. Y. TRANVouEz.

MoAlic, Laurent, Saint Jean Discalcéat (1279-1349) : sa vie, son ouvre, dir. J.C. CASSARD.

NERRIEC, Thierry, La vie religieuse à Concarneau (1830-1912), dir. M.-T. CLOITRE.

Nicolas, Mikaël, L'évolution politique de Douarnenez (1944-1989), dir. F. BOUTHILLON.

OlLIVIER, Nicolas, La conscription dans l'arrondissement de Morlaix (1807-1810), dir. J.-Y. CARLUER.

PESNEL, Laurent, Les mottes castrales de la moitié occidentale du Morbihan, dir. J.-C. CASSARD.

Petuaud-Letang, Alexandre, Les leçons politiques et militaires du conflit vietnamien à travers la guerre du Golfe, dir. F. BOUTHILLON.

PICARD, Vincent, Les ressortissants des nations ennemies internes dans le Finistère (1914-1920), dir. M.-T. CLOITRE.
POLARD, Olivier, Étude des monnaies du site de Monterfil à Corseul, ancienne capitale des Coriosolites, dir. J.-Y. ÉVEILLARD.

PRIGENT, Arnaud, Rostrenen durant la guerre 1939-1944, dir. J.-Y. CARLUER.

PRIGENT, Magali, Le métier d'historien et le pouvoir de la censure à la fin du XVI siècle ou l'étude comparée et commentée des éditions de 1583, 1588 et 1618 de l' "Histoire de Bretagne " de Bertrand d'Argentré, dir. J. KERHERVE.

Riou, Nathalie, La résurrection de Landévennec sous le signe du renouveau 1922-1965, dir. Y. TRANVOUEZ.

RiouAl, Mickael, L'activité maritime dans les quartiers de Brest et du Conquet de 1764 à 1770, dir. J. TANGUY.

ROCHER, Katell, La vie quotidienne et la violence, d'après les procédures criminelles de la sénéchaussée de Quimperlé de 1720 à 1770 , dir. F. RoudAuT.

Rolland, Pascal, La formation de la langue littéraire vannetaise (XVII -XVIII siècles.), dir. F. RoudAuT.

SAIS DU BRELL, Marc, Le corps des ingénieurs du Génie Maritime de 1765 à 1848, dir. M.-T. CLOITRE.

Salou, Fabrice, Éloquence et enjeu politique à Athènes : Eschyle et Démosthène 